\title{
Estudios en Labiatae VII. Salvia yukoyukparum, Nueva Especie y Primer Representante de la Sección Tomentellae en Colombia
}

\author{
José Luis Fernández-Alonso \\ Instituto de Ciencias Naturales, Universidad Nacional de Colombia, Apartado Aéreo 7495, \\ Bogotá D.C., Colombia. jlfernandeza@unal.edu.co
}

Resumen. Se describe e ilustra Salvia yukoyukparum Fernández Alonso (Labiatae), una nueva especie endémica de la Serranía de Perijá, Colombia, asignable a Salvia sect. Tomentellae (Epling) Epling, sección no registrada previamente en territorio colombiano. Se comenta la distribución conocida y el estatus de conservación y se discuten las posibles afinidades de esta especie. Se presenta una clave para separar las especies suramericanas de la sección Tomentellae.

Abstract. The new species Salvia yukoyukparum Fernández Alonso (Labiatae) is described and illustrated; it is endemic to the Serranía of Perijá, Colombia, and is assigned to Salvia sect. Tomentellae (Epling) Epling, a section not previously recorded from Colombia. Its known distribution and conservation status is commented upon, and the possible affinities of this species are discussed. A key for the South American species of Salvia sect. Tomentellae is presented.

Key words: Colombia, IUCN Red List, Labiatae, Perijá, Salvia subg. Calosphace, Salvia sections Flocculosae, Tomentellae.

El género Salvia L. con unas 930 especies, el más extenso de la familia Labiatae, cuenta en Colombia con 83 taxones reconocidos, siendo también el más diverso de las labiadas colombianas (Wood \& Harley, 1989; Fernández-Alonso et al., 2003; FernándezAlonso \& Rivera-Díaz, 2006). En la última década se adelantaron algunos trabajos en Salvia de Colombia que trataron diversas novedades taxonómicas y corológicas y algunos casos de hibridación natural, principalmente en las secciones Flexuosae (Epling) Epling, Longipes Epling, Rubescentes (Epling) Epling y Secundae (Epling) Epling (Fernández-Alonso, 1991, 1995a, b, c, 1996). Más recientemente, se dieron a conocer también para Colombia otras novedades de diversa índole en las secciones Angulatae (Epling) Epling, Macrostachyae (Epling) Epling, Purpureae (Epling) Epling, Siphonantha (Epling) Epling y Tubiflorae (Epling) Epling y se comentaron también algunos aspectos sobre fitogeografía de estos grupos
(Fernández-Alonso, 2002, 2003a, b, 2006). Todas las secciones mencionadas, así como el resto de las especies de Salvia nativas de Colombia, se adscriben al subgénero Calosphace (Bentham) Bentham.

En esta contribución se presenta la descripción de una especie nueva asignable a Salvia sect. Tomentellae (Epling) Epling, sección integrada por 20 especies: nueve de México y 11 de los Andes centrales (Ruiz \& Pavón, 1798; Epling, 1935-1937, 1939, 1947; Macbride, 1960; Epling \& Játiva, 1968; Ramamoorthy, 1984; Zarucchi, 1993; Orfila \& Farina, 1996; Harley, 1999). La sección Tomentellae no había sido registrada aún en territorio colombiano, y la única especie conocida del norte de Suramérica (Ecuador) S. humboldtiana F. Dietrich, es una planta de flores pequeñas y hojas enteras muy pequeñas, muy diferente a la que ahora se describe.

Salvia yukoyukparum Fernández Alonso, sp. nov. TIPO: Colombia. Cesar: Serranía de Perijá, Mpio. de Becerril, Corr. Siete de Agosto, entre la Cuchilla Machosolo y el Río Guajirita, 1500 m, 12 dic. 2005 (fl, fr), J. O. Rivera-Díaz, N. J. Garzón \& C. A. Vargas 2935 (holotipo, COL; isotipos, COL, HUA, MO, US). Figura 1.

Species Salviae sectioni Tomentellae (Epling) Epling tentatim adscribenda, sed ab omnibus speciebus sectionis indumento caulis, foliis (subtus) et calyce piliis ramosis praeditis, inflorescentiis spiciformibus, terminalibus vel axillaribus ad basin interruptis; bracteis lanceolatis vel ovato-lanceolatis, magnis (usque ad $6 \mathrm{~cm}$ longis), persistentibus, et corollae tubo ca. $15 \mathrm{~mm}$ longo, intus rugis binis in parte tertia distali positis differt.

Arbusto de hasta $1.8-2 \mathrm{~m}$, generalmente ramoso, de ramas escandentes, gráciles; tallos cuadrangulares, marrón pálido, de 2.5-4.5 mm diám., con indumento blanco, abundante, de pelos ramosos de hasta $1 \mathrm{~mm}$, enmarañado y regularmente distribuido en toda la superficie; entrenudos 2.5-6 cm. Hojas con pecíolo de 10-13 mm en la zona media e inferior del tallo y más corto en las superiores, con indumento ramoso de pelos septados, glandulares; láminas con haz verde y envés verde blanquecino o a veces (en las láminas viejas) con tinte morado, de consistencia membranosa, 

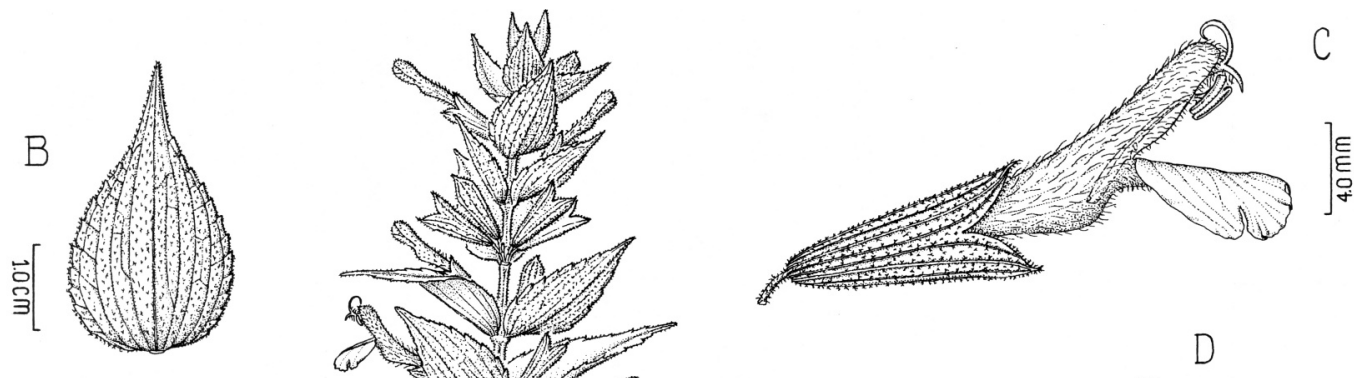

C E

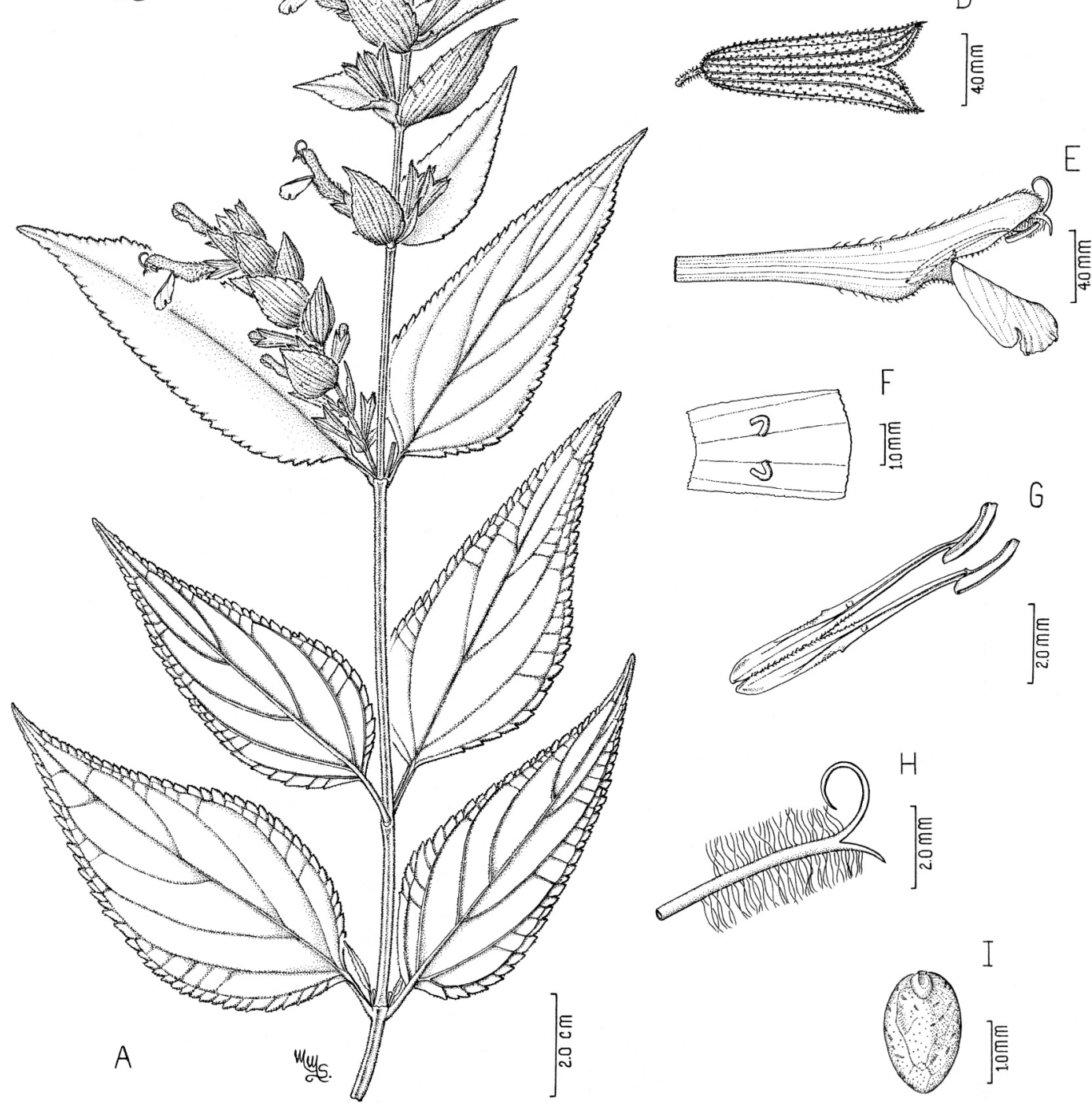

Figura 1. Salvia yukoyukparum Fernández Alonso. —A. Detalle de una rama con inflorescencia. —B. Bráctea de la zona media de la inflorescencia. - C. Flor. - D. Cáliz después de la antesis. - E. Corola mostrando la posición de estigmas y anteras. - F. Papilas dorsales (estaminodios), en la cara interna del tubo de la corola. — G. Conectivos estaminales con las anteras. - H. Estilo (parte distal) y estigmas. - I. Núcula. A partir del holótipo, Rivera-Díaz et al. 2935 (COL).

lanceoladas u ovado-lanceoladas, 6-10(13) $\times 2.5-$ $4.5 \mathrm{~cm}$, de base redondeada o truncada, ápice largamente acuminado y margen gruesa y agudamente dentado-aserrada, con 4 a 5 nervios laterales a cada lado del nervio medio y con nerviación de tercer y cuarto orden reticulada, poco resaltada; glabrescentes por la haz, con glándulas y protuberancias levemente resaltadas de aspecto cristalino; en el envés con pelos 
ramosos cortos, amarillentos (en seco) y también con glándulas sésiles. Inflorescencias terminales o laterales (axilares) espiciformes, interrumpidas en la base, de 10-15(20) cm de longitud; porción basal del raquis desnuda de 6-7 cm, seguida de 5 a 8(10) verticilastros; raramente con otras dos espigas laterales en la base del raquis; éste con indumento ramoso denso, de hasta $1 \mathrm{~mm}$; verticilastros acompañados de brácteas foliáceas persistentes, verdes o verde-morado, lanceoladas u ovado-lanceoladas, cóncavas, las inferiores de hasta $6 \times 2.5 \mathrm{~cm}$, las medias y superiores $2-4.5 \times$ $1.5-2 \mathrm{~cm}$, con margen gruesamente dentada, con nervio medio marcado y 2 a 3 nervios basales a cada lado; glabrescentes, con pelos muy finos y glándulas sésiles oscuras, excavadas; los primeros 1 a 3 verticilastros (inferiores), laxamente dispuestos, separados entre sí $2-3 \mathrm{~cm}$, el resto aproximadoimbricados, con 7 a 15 flores cada uno. Flores subsésiles, pedicelo floral de hasta $2 \mathrm{~mm}$ de longitud con indumento septado; cáliz verde o verde con tinte morado, 11-13 mm en la flor, y hasta $13.5 \mathrm{~mm}$ en el fruto, con labios subiguales, abiertos y cortos, subtriangulares, levemente acuminados, $2.5-3 \mathrm{~mm}$, con 7 nervios conspicuos en el labio superior; con indumento amarillento (en seco), corto, ramoso y densamente dispuesto; corola azul-morado, 20$22 \mathrm{~mm}$; tubo de ca. $15 \mathrm{~mm}$ de longitud, $2 \mathrm{~mm}$ de ancho en la parte basal y ensanchado-venticroso cerca de la boca del tubo, ésta de $3.5 \mathrm{~mm}$ de ancho, muy levemente arqueado-ascendente en el tercio distal; con dos papilas cintiformes (estaminoidales), arqueadas, de ca. $1 \mathrm{~mm}$, ubicadas hacia la mitad dorsal de la corola (10-11 cm por encima de la base del tubo); labio superior de $5 \mathrm{~mm}$, linear-espatulado, ligeramente galeado; labio inferior $5-6 \mathrm{~mm}$, superficie externa de la corola con pelos dispersos, rectos, tenues, algunos ramificados y glandulares (ramas con cabeza glandular); estambres ubicados en el tercio distal de la corola, con filamento de $1 \mathrm{~mm}$, conectivo levemente arqueado cerca de la antera, 3.5$4 \mathrm{~mm}$, alojado enteramente en el labio superior de la corola y en la boca del tubo; articulación del conectivo (porción inferior) de ca. $2 \mathrm{~mm}$; anteras oscuras, estrechamente cilíndricas, de $1.5 \mathrm{~mm}$, arqueado-convexas después de la dehiscencia, polen amarillo; estilo $22-23 \mathrm{~mm}$, con ramas estigmáticas desiguales, la superior más larga de $2-3 \mathrm{~mm}$ y fuertemente curvada hacia arriba formando un círculo, y la inferior dirigida hacia abajo, suberecta, de ca. $1 \mathrm{~mm}$, con denso indumento de pelos cintiformes agrupado-fasciculados en la parte distal, dispuestos en un solo plano. Núculas marrones, elipsoide-ovoides, con puntuaciones diminutas, dispersas y manchas difusas de color café oscuro, de aspecto ferrugíneo, 1.9-2.1 $\times 1.1-1.2 \mathrm{~mm}$, con abundante mucílago desecado de forma laminar (en algunas de ellas).

Hábitat y distribución. Hasta donde se conoce, se trata de una especie endémica de Colombia, de la Serranía de Perijá, en el ramal más norteño de la cordillera Oriental de Colombia. Se ha localizado entre 1400 y $1500 \mathrm{~m}$, en una franja de bosque subandino alterado, creciendo en taludes y pendientes expuestas, donde es localmente abundante.

Conservación. Salvia yukoyukparum es conocida hasta la fecha solo por una recolección (la serie típica), de una sola localidad. La asignación de esta especie a una sección que no cuenta con otros taxones en regiones cercanas (se concentra en México y en los Andes centrales) y el hecho de que no se haya localizado en los herbarios colombianos otro material proveniente de expediciones anteriores adelantadas en la Serranía de Perijá, asignable a la especie (Rivera-Díaz \& Fernández-Alonso, 2003), nos hace pensar que estamos ante un taxon endémico de distribución muy restringida en la actualidad. En este sentido, consideramos a S. yukoyukparum un taxon de carácter relíctico, en una sección que pudo tener una mayor presencia y distribución en el norte de Suramérica en épocas pasadas. De acuerdo con información reciente recabada en la localidad tipo, la región donde habita esta planta está sujeta a fuertes modificaciones, principalmente por fuego y limpia de vegetación secundaria, lo que impidió relocalizar los individuos vistos apenas 6 meses antes (O. Rivera, com. pers.). Por los factores mencionados: muy escasa extensión de presencia y alteración progresiva del hábitat conocido, pensamos que cabe su catalogación actual como especie amenazada según la categoría EN (En Peligro) de la IUCN (2001). Siguiendo los mismos parámetros utilizados para la categorización de la familia Labiatae en la serie Libros Rojos de las Plantas de Colombia (Fernández-Alonso \& RiveraDíaz, 2006), los criterios aplicados son los de areal pequeño y en disminución (EN Blabiii).

Etimología. El nombre de la especie hace referencia al pueblo indígena yuko-yukpa, de la familia lingüística Karib, también denominado tradicionalmente como pueblo "motilón", que tuvo y tiene en la región de Becerril, Cesar (Serranía de Perijá), alguno de sus asentamientos o resguardos (Jaramillo, 1992).

Discusión. Se asigna tentativamente Salvia yukoyukparum a la sección Tomentellae por tratarse de un arbusto provisto de pelos ramificados en tallos, hojas y cálices; labio superior del cáliz con siete nervios; corola azul, ventricosa en la mitad distal y con dos papilas cintiformes arqueadas en la cara 
interna en posición dorsal (infrecuente en la sección); estambres con porción inferior del conectivo recta y entera y estilo piloso en la zona distal. Aunque esta especie puede considerarse también afín en algunos caracteres a la sección Flocculosae (Epling) Epling (como son el indumento ramoso y las brácteas de la inflorescencia conspicuas y persistentes), la presencia de papilas en el tubo de la corola y las hojas y flores muy grandes para lo habitual en esta sección, permiten apartarla con claridad. Podemos separar con facilidad a $S$. yukoyukparum del resto de las especies conocidas de la sección Tomentellae, por la combinación de caracteres: connectivo estaminal subentero, estilo hirsuto en su región distal y brácteas de la inflorescencia persistentes, de más de $2 \mathrm{~cm}$ de longitud y corola morado-lila. Así mismo, S. yukoyukparum se diferencia sin dificultad del resto de las especies de Salvia conocidas en Colombia por presentar: indumento ramoso en tallos, hojas y cálices; brácteas de la inflorescencia amplias y persistentes; tubo de corola de con dos papilas ubicadas internamente en la mitad distal superior y estambres no exertos, de conectivo ligeramente arqueado.

\section{Clave Para las Especies Suramericanas de Salvia seccion TOMENTELLAE}

1a. Conectivo estaminal subentero, sin diente saliente... 2

1b. Conectivo estaminal con diente saliente . . . . . . 4 4

2a. Cáliz floral de 11-13 mm de longitud; estilo hirsuto. . .3

2b. Cáliz floral de 6-8 $\mathrm{mm}$ de longitud; estilo subglabro (Perú) . . . . . . . . . vargasii Epling

3a. Espigas cortas de 3-6 cm, con brácteas caducas de menos de $1 \mathrm{~cm}$, verticilastros separados $0.5-1 \mathrm{~cm}$; corola azul oscuro, tubo de la corola de ca. $20 \mathrm{~mm}$ (Bolivia). . . . . . . . . . . chorianthos Epling

3b. Espigas largas de 10-15(20) cm, con brácteas persistentes de más de $2 \mathrm{~cm}$, con verticilastros separados entre sí más de $1 \mathrm{~cm}$; corola azul-morado tubo de la corola de ca. $15 \mathrm{~mm}$ de longitud ( $\mathrm{N}$ de Colombia)....... S. yukoyukparum Fernandez Alonso

4a. Tubo de la corola de 13-14 mm de longitud (Perú) . . . . . . . . S. subscandens Epling \& Játiva

4b. Tubo de la corola de 3.5-12 mm . . . . . . . . 5

5a. Hoja entera, adelgazada en la base (Ecuador). . . . . . . . . . . . S. humboldtiana F. Dietrich

5b. Hoja aserrada, redondeada, truncada o cordada en la base . . . . . . . . . . . 6

6a. Haz suavemente cinereo-pubescente (Argentina) . . . . . . . . . . lorentzii Grisebach

6b. Haz verde, glabrescente. . . . . . . . . . 7

7a. Láminas de 8-12 × 2.5-4 cm; con 6 a 12 flores por verticilastro; con dos papilas en la mitad inferior interna del tubo de la corola (Argentina) $\ldots \ldots \ldots \ldots$. . . . . . calolophos Epling

7b. Láminas de $2-8 \times 1-3 \mathrm{~cm}$; con 3 a 6 flores por verticilastro; sin papilas en la cara interna del tubo de la corola . . . . . . . . . . . . . . 8

8a. Lámina foliar de $2-4.5 \mathrm{~cm}$; tubo de la corola de $4.5-6.5 \mathrm{~mm} \ldots \ldots \ldots \ldots \ldots$. . . . . . . 9 8b. Lámina foliar de (4)4.5-8 cm; tubo de la corola de 8-12 mm (Bolivia) . . . . . . . . . . . 10

9a. Labios del cáliz de 1-1.5 mm, subespinulosos en el ápice (Perú) . . . . . . S. cuspidata Ruiz \& Pavón

9b. Labios del cáliz de 3-7 mm, redondeado-mucronados en el ápice (Bolivia, Argentina, Chile). . . .................... gilliesii Bentham

10a. Envés foliar blanco, finamente tomentoso; cáliz maduro de 11-12 mm; tubo de la corola de $12 \mathrm{~mm} . \ldots \ldots \ldots \ldots \ldots \ldots$. retinervia Briquet

10b. Envés foliar esparcidamente pubescente y con glándulas; cáliz maduro de $8 \mathrm{~mm}$; tubo de la corola de $8-10 \mathrm{~mm}$. . . . . . . . S. bangii Rusby

Agradecimientos. A Orlando Rivera que recolectó la serie típica aquí descrita en el marco de un reciente estudio de la vegetación del sector Centro-Norte de la Serranía de Perijá y facilitó el acceso a las muestras. Al antropólogo Gaspar Morcote por su valiosa información sobre la comunidad yuko-yukpa de Perijá. A la Universidad Nacional de Colombia y al Herbario Nacional Colombiano (COL), por las facilidades dadas para el desarrollo del presente trabajo. A G. Orsini, M. Foley y V. Hollowell, por las valiosas observaciones al manuscrito.

\section{Literatura Citada}

Epling, C. 1935-1937. Synopsis of the South American Labiatae. Repert. Spec. Nov. Regni Veg. Beih. 85: 1341.

1939. A Revision of Salvia subgenus Calosphace. Feddes Repert. Spec. Nov. Regni Veg. Beih. 110

. 1947. Supplementary notes on American Labiatae IV. Bull. Torrey Bot. Club 74(6): 512-518.

\& C. Játiva. 1968. Supplementary notes on American Labiatae X. Brittonia 20: 295-313.

Fernández-Alonso, J. L. 1991. Dos nuevos híbridos en Salvia (Labiatae) con potencial ornamental. Trianea 4: 329-340. . 1995a. Una nueva especie de Salvia (Labiatae) de Colombia. Anales Jard. Bot. Madrid 52(2): 159-162.

- 1995b. Estudios en Labiatae de Colombia I. Novedades en los géneros Salvia e Hyptis. Revista Acad. Colomb. Ci. Exact. 19: 469-480.

1995c. Estudios en Labiatae de Colombia II.

Novedades en Salvia sect. Longipes Epling. Anales Jard.

Bot. Madrid 53(1): 41-46.

. 1996. Labiadas: La familia de la menta, la salvia y el mastranto. Pp. 154-156 en L. Montes \& P. Eguiluz (editors), El Cerro, Frontera Abierta. Recorrido Ecológico por el Cerro de Usaquén. Edit. Santillana, Bogotá.

2002. Estudios en Labiatae de Colombia III. Novedades en Lepechinia Willd., Salvia L. y Satureja L. Anales Jard. Bot. Madrid 59(2): 344-348.

- 2003a. Estudios en Labiatae de Colombia IV.

Novedades en Salvia y sinopsis de las secciones Angulatae y Purpureae. Caldasia 25(2): 235-281.

- 2003b. Algunos patrones de distribución y endemismo en plantas vasculares de los páramos de Colombia. Pp. 213-240 en Memorias Congreso Mundial de Páramos, Tomo I. Ministerio de Medio Ambiente. Paipa, Colombia.

- 2006. Revisión taxonómica de Salvia sect. Siphonantha (Labiatae). Anales Jard. Bot. Madrid 63(2): 145-157. 
\& O. Rivera-Díaz. 2006. Labiatae. Pp. 385-582 en N. García \& G. Galeano (editors), Libro Rojo de las Plantas de Colombia 3: Las Bromelias, las Labiadas y las Pasifloras. Serie Libros Rojos de Especies Amenazadas de Colombia, Bogotá, Colombia.

, N. Vega, J. J. Filgueira \& G. Pérez. 2003. Lectin prospecting in Colombian Labiatae. A systematic-ecological approach. Biochem. Syst. Ecol. 31(2003): 617-633.

Harley, R. M. 1999. Labiatae. Pp. 519-526 en P. M. Jorgensen \& S. León-Yañez (editors), Catálogo de las Plantas Vasculares del Ecuador. Monogr. Syst. Bot. Missouri Bot. Gard. 75.

IUCN. 2001. IUCN Red List Categories and Criteria, Version 3.1. Prepared by the IUCN Species Survival Commission. IUCN, Gland, Switzerland, and Cambridge, United Kingdom.

Jaramillo, G. O. 1992. Geografía humana de Colombia: Nordeste indígena, Tomo 2. Instituto Colombiano de Cultura Hispánica, Santafé de Bogotá, Colombia.
Macbride, J. F. 1960. Labiatae. Pp. 721-829 en J. F. Macbride (editor), Flora of Peru. Field Mus. Nat. Hist., Bot. Ser. 13, Part 5(2).

Orfila, E. N. \& E. L. Farina. 1996. Lamiaceae-Flora del Valle de Lerma. Aportes Bot. Salta. Ser. Fl. 4(2): 1-67.

Ramamoorthy, T. P. 1984. Notes on Salvia (Labiatae) in Mexico, with three new species. J. Arnold Arbor. 65: 135-143.

Rivera-Díaz, O. \& J. L. Fernández-Alonso. 2003. Análisis corológico de la flora endémica de la Serranía de Perijá, Colombia. Anales Jard. Bot. Madrid 60(2): 347-369.

Ruiz, H. \& J. Pavón. 1798. Flora Peruviana et Chilensis I. Tipogr. G. de Sancha, Madrid.

Wood, J. R. I. \& R. M. Harley. 1989. The genus Salvia in Colombia. Kew Bull. 44: 211-279.

Zarucchi, J. L. 1993. Labiatae. Pp. 579-590 en L. Brako \& J. L. Zarucchi (editors), Catálogo de las Angiospermas y Gimnospermas del Perú. Monogr. Syst. Bot. Missouri Bot. Gard. 45. 